\title{
Thyroid Homeostasis and Inflammation, the Physiological Changes of Exercise of Thermogenesis
}

\author{
Ronaldo Arkader ${ }^{1 *}$, Rosa $\mathrm{MR}^{2}$ and Moretti $\mathrm{G}^{2}$ \\ ${ }^{1}$ Department of Endocrinology and Sports Medicine, Brazil \\ ${ }^{2}$ Anhembi Morumbi University, Brazil
}

Submission: June 06, 2017; Published: June 28, 2017

*Corresponding author: Ronaldo Arkader MD, PhD, Endocrinologist and Sports Medicine, Brazil, Email: r-arkader@uol.com.br

\begin{abstract}
The human body when subjected to heavy loads of physical training suffers numerous modifications to adapt to stress. Various systems are directly and indirectly affected, we can mention the cardiorespiratory system as the one that suffers the most modifications when we are facing an.intense physical load. Another system that modifies and of which we will explain in this paper is the endocrine system, specifically the thyroid gland who suffers some changes. This paper aims to demonstrate and exemplify the changes in the thyroid gland during physical activity, such as the release of pro- and anti-inflammatory cytokines, and the alteration occurred in the release of thyroid hormones. All these changes suffer direct influence on the athlete's performance being a limiting factor for a good performance. Through this paper, we exemplify the changes in the thyroid gland during exercise in order to seek an intervention for these transformations, aiming ultimately a good performance of the athlete.
\end{abstract}

\section{Introduction}

Thyroid Hormones (TH) act on multiple metabolic process influencing the concentration and activity of numerous enzymes, the metabolism of substrates, vitamins and minerals, and the response of target tissues to several hormones. These TH have critical roles in cell differentiation, growth, and regulation of oxygen consumption and thermogenesis [1]. Thyroid disease occurs when the gland is enlarged (goiter) or there are alterations in the secretion of triiodothyronine (T3) or thyroxin (T4). The thyroid gland secretes both $\mathrm{T} 3$ and T4, but it only is responsible for approximately $20 \%$ of $\mathrm{T} 3$ secretion and is the only endogenous source of T4. The remainder of T3 in the body is the breakdown product of the peripheral conversion of T4 to T3. These TH are part of a negative feedback loop with the anterior pituitary gland. Negative feedback is seen when the output of a pathway inhibits inputs to the pathway controlling the release of thyroid stimulating hormone in response to circulating levels of TH.

The release of thyroid releasing hormone (TRH) by the hypothalamus stimulates the release of TSH by the anterior pituitary. TSH binds to epithelial cells on the thyroid gland stimulating the release of TH (T3 and T4). When the concentration of these hormones reaches a threshold, the TRH secretion is inhibited. Thyroid disorders include insufficient TH secretion (hypothyroidism) and increased secretion (hyperthyroidism.).
As reported in a systemic review the estimated incidence of hypothyroidism in women is $350 / 100,000$ and in men $80 / 100,000$, hyperthyroidism's incidence is $80 / 100,000$ in women and 8/100,000 in men. These values reflect a twofold increase from previous estimates, which may represent an increase in the overall incidence of autoimmunity. TH are important regulators of cardiac function and myocardial arteriolar density [2].

Just as the increased incidence of thyroid disease is a current finding, has increased annually the number of participants in physical activities of high performance (marathons, iron lifting, triathlon), leading the body to release pro-inflammatory substances in an acute form. We know of the existence of subclinical hypothyroidism and that these inflammatory markers inhibit peripheral conversion of $\mathrm{T} 4$ to $\mathrm{T} 3$, and it increases the peripheral conversion of $\mathrm{T} 4$ to reverse $\mathrm{T} 3$. There are not many studies in athletes with hypothyroidism, and the treatment may unfortunately decrease the performance of the athlete. Our aim is to review some concepts and evidence in the medical literature.

\section{Inflammation and Exercise}

The role of inflammation in skeletal muscle adaptation to exercise is complex and has hardly elucidated so far. While the acute inflammatory response to exercise seems to promote 
skeletal muscle training adaptation and regeneration, persistent low-grade inflammation, as seen in a multitude of chronic diseases, is obviously detrimental. Lately the studies of the regulation of cytokine production in skeletal muscle cells has been clarified, yet little is known about the compensatory and anti-inflammatory mechanisms that resolve inflammation and restore tissue homeostasis. One important strategy to ensure sequential, timely and controlled resolution of inflammation relies on the regulated stability of mRNAs encoding proinflammatory mediators [3].

Sedentary lifestyle is a risk factor and a strong predictor for chronic disease and premature death. Low-grade inflammation it is a role player in the pathogenesis of cardiovascular disease. Inflammatory process has been also involved in maintaining the balance between coagulation and fibrinolysis. In addition, an inverse linear dose -response relation between physical activity and mortality risks it was reported. However, the favorable effects of structured exercise programs and the independent contribution of physical activity to cardiovascular risk are still under investigation. The coordination, amplification, regulating, magnitude and duration of the inflammatory events and, therefore, its effects are due to a group of glycoproteins, the cytokines. Cytokines are proteins secreted by cells of the innate and adaptive immunity, which mediate many functions of these cells; their production is in response to microorganisms and other antigens. Different cytokines stimulate various responses of the cells involved in immunity and inflammation and is often referred as biological response modifiers. Cytokines can be functionally divided into three categories:

a. Mediators and regulators of innate immunity (TNF, IL1, IL12, IL10, etc.)

b. Mediate adaptive immunity (IL2, IL4, etc.) and

c. Stimulating hematopoiesis (IL7, IL3, etc.).

The lesion in muscle tissue induced by exercise can signal through cytokines. Other tissues and cells of the endocrine and immune systems, particularly the hypothalamic-pituitaryadrenal axis and hypothalamic-pituitary -gonadal promote a combined action necessary for its repair. The balance between the actions of different cytokines pro and anti-inflammatory contributes to the complete repair of damaged tissues. In the cascade of cytokines after a stress (e.g., exercise or infection), the initial cytokines appear in the following order: TNFa, IL1, IL6 receptors of IL1ra cytokines (inhibitor of IL1) and sTNF-R (order TNFa inhibitor). Therefore presence of infection or acute stress in many of these cytokines and their receptors are multiplied and when the effect of stress or infection ends, generally, the plasma levels of these cytokines decreases [4].

The major pro -inflammatory cytokines, which are "alarm cytokines" include interleukin (IL-1 $\beta$ ) and TNF. Exercise appears to attenuate the basal levels of rest TNF. A transversal cohort study using level of self-reported physical activity showed that people with high levels of physical activity had circulating levels of TNFa and other inflammatory markers (such as IL6 and reactive protein $\mathrm{C}$ ) lower than those who self-reported sedentary. These data indicates the level of physical activity alone relates inversely with circulating levels of TNFa. In fact, studies show that the high level of physical activity decreases the levels of mediators of peripheral inflammatory response, including TNFa, at a rate of $20-60 \%$ compared to sedentary lifestyle. Interleukin 1 is a pro-inflammatory cytokine and the acute physical exercise usually does not raise your concentration. This can be partly explained because of physical activity increases the levels of IL1ra (receptor antagonist IL-1) which, in conformation, can bind to this cytokine, yet, IL1ra does not make signal transduction to IL1 to act, leaving then this interleukin disabled. However, some studies have shown that chronic physical activity appears to decrease the plasma levels of this cytokine. A known manner of prevention of all these diseases is the regular physical activity. The first cytokines in the cascade are TNFa and IL-1 pro -inflammatory cytokines, after the continuation of the cascade arrived IL6, taken as pro- and anti-inflammatory, and after that, following the release of IL1ra and sTNF-R classified as factors anti-inflammatories. Generally, after acute exercise there is no increase in pro-inflammatory cytokines (IL-1 and TNFa). This can be partly explained by increased IL-6, which induces the synthesis of antagonistic receptor of IL-1 and TNFa (IL1ra and STNF-R, respectively) and other anti-inflammatory cytokines [4].

However, regardless of IL6, exercise by itself can only remove other ways from entry of these pro-inflammatory cytokines in plasma. Chronic exercise generally acts positively on all mentioned cytokines. With regard to TNF and IL-1 acts mainly in the reduction of plasma levels as physical activity becomes chronic. In the acute stage of the exercise these cytokines do not seem to increase, due to the increases of its inhibitory receptors (sTNF-R and IL1-ra), acting in this way, an important role in the prevention and treatment of non-transmissible chronic diseases. IL-6 in the acute phase increases both as aerobic exercises as in strength exercise. This significant increase has a biological explanation, since through it can induce the synthesis of antiinflammatory factors, such as TNF-R, IL1ra and IL10, possible damage shields caused by excessive pro-inflammatory cytokines in blood circulation. Chronically, both the exercise of strength and aerobic seem to decrease your basal plasma levels [4].

By contrast, regular exercise and physical activity are protective against all-cause mortality through suppressing proinflammatory cytokine production, enhancing anti-inflammatory mediators and antioxidant development and promoting fibrinolytic activity. Low-load resistance exercise also plays an advantageous role in thrombogenesis by reducing inflammatory processes and potentiating fibrinolytic characteristics. Physical activity is well known as an important strategy not only to prevent, but also to improve and-in some cases-even cure chronic inflammatory disease conditions. 
Excess physical training as much as psychological stress, can promote a change in the hormonal balance, and this fact is associated with over training. At the same time, it appears that the high release of pro-inflammatory cytokines triggered by systemic inflammation process resulting from over training, acts on the central nervous system. The receptors for IL-1 and IL- 6 cytokines in the brain are abundant in the hypothalamic region. The interaction of these cytokines to specific receptors in paraventricular hypothalamic nuclei results in the release of corticotrophin-releasing hormone ( $\mathrm{CRH}$ ) and, consequently, ACTH and cortisol. Allied with the action of cytokines in the hypothalamus, IL- 6 can control the release of steroid hormones by direct action on the adrenal cells and regulate the synthesis of mineralocorticoids, glucocorticoids and androgens. This control is dependent on the concentration and duration of exposure to IL-6. Thus, systemic inflammation and elevated serum levels of cytokines may be responsible for increasing the serum cortisol levels observed in subjects with over training. Moreover, the increased concentration of IL- $1 \beta$ and IL- 6 can promote the activation of different hypothalamic nuclei, which may account for many of the behavioral changes related to illness such as reduction in appetite, depression, commonly seen among athletes with over training.

\section{Thyroid Disordes in Athletes}

The muscle cells obtain the energy they need for their contraction by two mechanisms: anaerobic and aerobic. The anaerobic mechanism is the one that provides energy without oxygen consumption, although it is the first activated and is very brief. On the other hand, in the aerobic mechanism, muscle cells obtain energy from the use of oxygen absorbing from blood circulation yielding a residue of carbon dioxide that passes into the blood being eliminated by the lungs. The aerobic mechanism only happens about forty seconds after the beginning of the exercise in which is predominant very prolonged effort or resistance.

The muscle fibers usually have the necessary oxygen supplement for their activity, forty seconds after the start of exercise thanks to a series of changes produced in the functioning of the cardiorespiratory system. This set of changes controlled by the autonomic nervous system and mediated by various hormones is precisely called as "cardiorespiratory adaptation to exercise." The first modification corresponds to the increased amount of blood pumped by the heart to the vascular system. The increased volume of blood ejected by the ventricle has an impact on the large arteries. The fact that its walls are subjected to a higher pressure providing another consequent changes in cardiac adaptation to physical exercise, i.e. the increasing of the maximum blood pressure, which at rest is around $120 \mathrm{~mm} \mathrm{Hg}$, during physical exercise, may rise to 160 or $200 \mathrm{~mm} \mathrm{Hg}$.

Another fundamental change in cardiorespiratory adaptation to exercise is the redistribution of blood flow to the body. This mechanism produced by expansion and contraction of the arteries of various organs has the mission to increase oxygen transport to the tissues, subjecting it to a greater effort. In this case, the skeletal muscles and the heart itself, reduces the oxygen uptake of tissue not involved in the exercise. Another important part of cardiorespiratory adaptation to physical exercise corresponds to the airways, which also modify its operation so as to ensure a greater flow of oxygen into the lungs and a greater removal of carbon dioxide to the outside, increasing the respiratory rate and gas exchange in the lung alveoli. This cardiorespiratory adaptation during physical exercise usually is followed by increasing the oxygen absorbing capacity of muscle cells from the bloodstream and carbon dioxide elimination rate in the opposite direction.

The adaptation by the cardiovascular system to the exercise is in the medium and long term beneficial to improve the performance of the heart and lungs, and can prevent and even treat some serious problems that could affect these organs. Either way the benefits are only effective when the resistance exercise is performed and done regularly, with moderation and progressively. On the other hand, another system that suffers modifications when subjected to physical exercise is the endocrine system specifically the thyroid gland.

Significant changes in energy metabolism occur during exercise and remains for a few hours after revealing challenges for metabolic homeostasis. Exercise corresponds to a physical stress followed by some endocrine modifications that occur in order to counterbalance its effects on thermogenesis and substrate metabolism [1]. Regulation of the metabolic adaptation during prolonged physical training by the thyroid hormones it is important but remains controversial. Some authors reported no major effect of exercise training on serum TSH levels in professional athletes and sedentary individuals, suggesting that the influence of chronic exercise is likely to be small. In contrast, other authors suggested long -term exercise training might lead to down regulation of thyroid hormone concentrations. Among the factors, this response by the exercise induces negative energy balance as well as signaling alterations in the hypothalamuspituitary-thyroid and hypothalamus- adipocyte-leptin axes [5].

Exercise is a stressful situation that challenges body homeostasis, so that the organism has to reestablish a new dynamic equilibrium in order to minimize cell damage. One of the systems affected is the hypothalamic-pituitary-thyroid axis. In athletes with thyroid disorders, a decrease in their performance usually is the presenting complaint to the clinician. This may elucidate suspicion of a hormonal cause. The signs may not be as pronounced in this population of patients, and the physician must use his clinical judgment in the laboratory workup. A retrospective report of 50 athletes with complaints of fatigue concluded that the yield from a selection of blood tests, including $\mathrm{TSH}$, was low. In this case, only three abnormal laboratory results (6\%) were found that might have contributed to the diagnosis of medical disease as a cause for fatigue. Furthermore, abnormal 
TSH values were not implicated as a cause [2]. Hyperthyroidism and hypothyroidism were associated with all-cause and cardiovascular mortality in many, but not all, previous studies. Several Meta-analyzes have been published on this matter so far with conflicting results, although more recently studies has shown that both subclinical hyper- and hypothyroidism were positively associated with all cause and cardiovascular mortality [6]. This study aims to show the importance of the control of thyroid hormone in athletes high and low performance, whereas the thyroid dysfunctions are associated with cardiovascular mortality in addition to affect the athlete's performance.

According to some authors, TSH secretion possibly increases during exercise. This stimulation is elucidated by the body's need to increase its metabolism to practice exercise. TSH levels remains elevated for several days after a competition and promotes a late increase in the release of T3 and T4. According to authors, prolonged submaximal exercise sessions induces the T4 to remain 35\% higher than the resting levels after an initial peak at the beginning of the exercise and T3 levels tend to increase as well. Already according to another author, there is greater deionization of T3 during exercise, however serum levels do not change $[7,8]$.

A study observed the variation of TSH, T4 and T3 after 30 minutes of swimming athletes in three different water temperatures $\left(20^{\circ} \mathrm{C}, 26^{\circ} \mathrm{C}\right.$ and $\left.32^{\circ} \mathrm{C}\right)$. This study that the TSH rose to $20^{\circ} \mathrm{C}$, kept at $26^{\circ} \mathrm{C}$ and decreased to $32{ }^{\circ} \mathrm{C}$. This may be due to the need for more energy in colder conditions. The free T4 (FT4) athletes had a similar response to TSH, increasing to 20 and $26{ }^{\circ} \mathrm{C}$ and decreasing to $32{ }^{\circ} \mathrm{C}$. Already T3 showed no significant changes after the three sets of exercise $[7,9]$.

In another study, male athletes have an increased secretion of T4 and degradation compared to sedentary individuals; however, the free T4 does not change with exercise. As for women athletes, when performing endurance training the thyroid function decreases, with lower levels of T3 and TSH hyper responsiveness to TRH stimulation. Another factor is that female athletes with excessive weight loss and amenorrhea by chronic stress caused by exercise can have a suppression of thyroid hormones, which can lead to low T3 syndrome, where there is a predominance of T3r. One hypothesis proposed to explain this mechanism is through NF-kappaB activation by norepinephrine, resulting in inactivation of the enzyme gene expression T3-dependent 5 '-deidodinase $[8,10,11]$.

An author presented a study of athletes in cycle ergometer exercise in which batteries were performed at different intensities of maximum heart rate (MHR): low (45\%), moderate $(70 \%)$ and high (90\%). Every three minutes was collected blood samples for measurement of T4, T3, free T4, free T3 and TSH, and the intensity increased. The maximum increase of all TSH extract hormones was observed in the aerobic threshold level $(70 \%$ of MHR). TSH increased almost linearly during the three sections. The T4 and free T4 increased significantly in submaximal intensity compared to $45 \%$. Already T3 and free $\mathrm{T} 3$ increased to $70 \%$, but decreased to $90 \%$, reaching almost the same level observed at $45 \%$ of maximum heart rate. TSH levels remained high by 15 minutes subsequent to the exercise, it perhaps to increases the levels of thyroid hormones needed during exercise [11].

According to studies, physical exercise is a physical stress, which alters homeostasis in response to this effect; the autonomous system reacts and participates to maintain the equilibrium of the human body. However, physical conditioning is associated with a decrease in the hypothalamic response to exercise, as demonstrated. A study of men with six months of endurance training, reported that $\mathrm{T} 4$ and free $\mathrm{T} 4$ concentrations were slightly decreased, with no change in TSH. Another study reported the effects of a week of intense strength training on thyroid hormones in weight lifters, where a decrease in TSH was demonstrated, T3 and T4, while free T4, rT3 and TBG were unchanged $[10,12]$.

We may note that all the authors agree that there are changes in serum levels of thyroid hormones during an exercise session. This observation is due to the fact that higher energy is required from the cells during activity but many also agree that thyroid activity decreases with training, perhaps for body adaptation.

\section{Discussion}

Due to the increased incidence of thyroid disease, it is extremely important to monitor the function of the thyroid as physical exercise can lead to a systemic inflammatory response and a dysregulation of the gland. To prevent this disorder, monitoring gland for effective control of metabolism is necessary. There are reports of control of thyroid hormones in the regulation of cardiac function, as an important factor in the control and prevention of diseases of the circulatory system. Thyroid function is a new step to the discovery of great champions, since thyroid function is directly related to the athlete's performance, that is, increasingly necessary to control the thyroid gland given that the athlete must achieve greater performances in their competitions.

Still in heavy exercise may be associated with a systemic inflammatory response with the release of interleukin-6 (IL-6) by contracting skeletal muscles, Followed by an acute reactant release of C-reactive protein (CRP). Both CRP and IL-6 can stimulate monocyte tissue factor production, provoke platelet hyper reactivity, promote fibrinogen biosynthesis, and enhance micro particle formation and erythrocyte binding, thus triggering prothrombotic state. This systemic inflammatory response can promote a change in the hormonal balance. At the same time, it appears that the higher releases of pro-inflammatory cytokines triggered by systemic inflammation process resulting from overtraining acts on the central nervous system. It can be said, that the thyroid gland when exposed to high load exercise may suffer dysregulation and thus compromising its functioning, 
once the inflammatory response generated by intense physical exercise causes a hormonal imbalance. The thyroid gland plays a key role during and after physical activity and its control is of utmost importance to the proper functioning of metabolism. These hormones have critical roles in cell differentiation, growth, and regulation of oxygen consumption and thermogenesis.

\section{Conclusion}

Based on studies regarding the activity of the thyroid gland when submitted to some stress, such as physical exercise, it is possible to conclude that physical activity when done regularly can reduce the basal levels of pro and anti -inflammatory cytokines. TNF is a cytokine that show a decrease in their levels compared to sedentary people, as well as with the levels of C-reactive protein. Another substance, which we can mention include interleukin 1, a pro-inflammatory cytokine character and not rises during physical activity. We conclude that regular physical activity is a protective factor for numerous diseases by decreasing the production of pro-inflammatory cytokines, increasing anti-inflammatory mediators and developing antioxidants promoting fibrinolytic activity. Physical activity is an important strategy not only for prevention but as a therapeutic form of chronic inflammatory diseases that threaten to become the great challenge of medicine such in its prevention and cure, seen the increase number of overweight and sedentary population who develop these disorders. The thyroid gland, responsible for the secretion of thyroid hormone is also affected when it is subjected to intense physical exercise. We can see that it is still contradictory if the physical exercise alters the levels of thyroid hormone, but some authors reported no significant effect of physical training on the levels of TSH, T3 and T4 in professional athletes and sedentary individuals suggesting that the influence of physical exercise is little. In contrast, other authors suggested long-term exercise training might lead to downregulation of thyroid hormone concentrations. Among the factors, this response by exercise induces negative energy balance as well as signaling alterations in the hypothalamuspituitary-thyroid and hypothalamus -adipocyte-leptin axes.

It still controversial, can't being evaluated for sure which are the effects of exercise on thyroid function, due to this, more experimental studies to seek changes in thyroid hormones during exercise are needed. In addition, papers should not be done with different types of exercise, since the effort involved and the environment can dramatically change the search result. We note that all authors agree that there are changes in serum levels of thyroid hormones during an exercise session. This observation is because there are major energy requirement from the cells during the activity yet many also agrees that thyroid activity decreases with training, perhaps to adapt the body.

\section{References}

1. Fortunato R, Ignacio D, Padron A, Pecanha R, Marassi M, et al. (2008) The effect of acute exercise session on thyroid hormone economy in rats. J Endocrinol 198(2): 347-353.

2. Duhig TJ, McKeag D (2009) Thyroid Disorders in Athletes. Curr Sports Med Rep 8(1): 16-19.

3. Beiter T, Hoene M, Prenzler F, Mooren FC, Steinacker JM, et al. (2015) Exercise, skeletal muscle and inflammation: ARE-binding proteins as key regulators in inflammatory and adaptive networks. Exerc Immunol Rev 21: 42-57.

4. Gonzaga Teodoro B, Costa Moreira O, Gouveia Peluzio M, José Natali A (2010) Resposta das citocinas ao exercício físico. Buenos Aires Año 15(144).

5. Perseghin G, Lattuada G, Ragogna F, Alberti G, La Torre A, et al. (2009) Free leptin index and thyroid function in male highly trained athletes. Eur J Endocrinol 161(6): 871-876.

6. Zhang Y, Chang Y, Ryu S, Cho J, Lee W, et al. (2014) Thyroid Hormones and Mortality Risk in Euthyroid Individuals: The Kangbuk Samsung Health Study. J Clin Endocrinol Metab 99(7): 2467-2476.

7. Canali ES, Kruel LFM (2001) Respostas hormonais ao exercício. Rev Paul Educ Fís 15(2): 141-153.

8. Pardini DP (2001) Alterações Hormonais da Mulher atleta. Arq Bras Endocrinol Metab 45(4): 343-351.

9. Deligiannis A, Karamouzis M, Kouidi E, Mougios V, Kallaras C (1993) Plasma T SH, T3, T4 and cortisol responses to swimming at varying water temperatures. Br J Sports Med 27 (4): 247-250.

10. Mastorakos G, Pavlatou M (2005) Exercise as a Stress Model and the Interplay Between the Hypothalamus-pituitary-adrenal and the Hypothalamus-pituitary-thyroid Axes. Horm Metab Res 37(9): 577584 .

11. Ciloglu F, Peker I, Pehlivan A, Karacabey K, Ilhan N, et al. (2005) Exercise intensity and its effects on thyroid hormones. Neuro Endocrinol Lett 26(6): 830-834.

12. Alén M, Pakarinen A, Häkkinen K (1993) Effects of prolonged training on serum thyrotropin and thyroid hormones in elite strength athletes. J Sports Sci 11(6): 493-497.

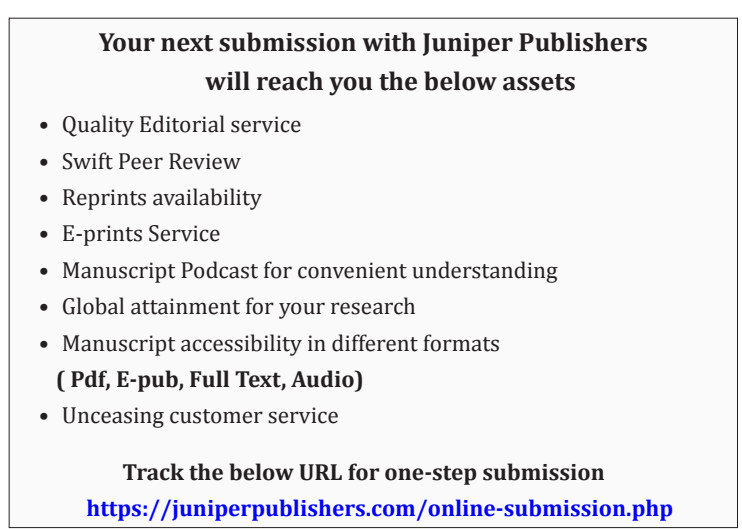

